\title{
Nonlinear mixing and mode correlations in a short Raman fiber laser
}

\author{
I.D. Vatnik ${ }^{* a}$, O. A. Gorbunov ${ }^{\mathrm{a}, \mathrm{b}}$, D. V. Churkin ${ }^{\mathrm{a}, \mathrm{b}, \mathrm{c}}$ \\ anstitute of Automation and Electrometry SB RAS, ac. Koptyug ave. 1, Novosibirsk, 63009, Russia; \\ ${ }^{\mathrm{b}}$ Novosibirsk State University, Pirogova str. 2, Novosibirsk 630090, Russia \\ ${ }^{c}$ Aston Institute of Photonic Technologies, Aston University, Birmingham, B4 7ET, United \\ Kingdom.
}

\begin{abstract}
In the present paper we experimentally demonstrate a generation in a short Raman fiber laser having 10000 different longitudinal modes only. We design the laser using 12 meters of commercially available fiber. Contrary to the recently demonstrated single longitudinal mode DFB Raman laser and short DBR Raman laser, in the laser under study the number of modes is high enough for efficient nonlinear interactions. Experimentally measured time dynamics reveals the presence of mode correlations in the radiation: the measured extreme events lasts for more than 10 round-trips.
\end{abstract}

Keywords: Raman laser, fiber laser, mode-locking

\section{INTRODUCTION}

Raman fiber lasers are usually based on long cavities having typical lengths from hundreds of meters to several kilometers. Ultra-long Raman fiber lasers (RFLs) have been demonstrated with a record cavity length up to $270 \mathrm{~km}^{1}$ being promising for telecom applications as quasi-lossless transmission media ${ }^{2-3}$. But most efficient and powerful RFLs proved to be based on shorter fiber spans. Hundred-watts level generation demonstrated recently in one hundred ${ }^{4}$ and thirty meters ${ }^{5}$ RFLs. In the case special fibers with a high Raman gain are used, it's possible to decrease threshold powers and obtain a generation in shorter span of $10 \mathrm{~m}^{6}$. In the paper ${ }^{7}$ high-efficiency polarized generation with up to 23 $\mathrm{W}$ and $0.4 \mathrm{~nm}$ width at $1178 \mathrm{~nm}$ was achieved in Raman "master oscillator power amplifier" scheme, with the Raman master oscillator cavity of only $10 \mathrm{~m}$.

Length shortening may also result in decrease of generation linewidth. Long (hundreds of meters) RFLs are characterized by a broad generation spectrum (a typical spectrum width is about $1 \mathrm{~nm}$ ) influenced by strong nonlinear spectral broadening via four-wave mixing of different longitudinal modes ${ }^{8},{ }^{9}$. The resulting number of generated modes could be very high - up to hundreds of millions, being proportional to the spectral width and inversely proportional to the cavity length. To achieve a narrow-band generation in Raman fiber lasers, DBR (distributed Bragg reflector) and DFB (distributed feedback) configurations are used recently, and only few longitudinal modes operation ${ }^{10}$ and even single mode operation ${ }^{11}$ have been demonstrated.

Mode interactions leading to specific time dynamics of the quasi-CW fiber lasers are of great interest In particular, the pulsed operation can be achieved via controlling the phase relations between different modes as it has been recently shown in RFLs using semiconductor saturable absorber mirror ${ }^{12}$ or carbon nanotubes ${ }^{13}$. Time dynamics in free-running Raman lasers, when no special measures are taken to lock phased of the modes, may differs considerably from both single-mode lasers operating in CW regime and mode-locked lasers, and depends on the amount of stochasticity in phase relations between different modes. In this case, even more interesting physics of weakly correlated modes is anticipated, like long-living coherent structures in output radiation ${ }^{14}$.

However, to study mode dynamics and inter-mode interactions, the relatively high number of generation modes is required. At the same time, the number of modes should not be as high as in conventional RFLs. Indeed, conventional long RFLs of broad spectrum generates much more longitudinal modes that could be numerically modeled.

*ilya.vatnik@gmail.com

Nonlinear Optics and Its Applications VIII; and Quantum Optics III, edited by Benjamin J. Eggleton, Alexander L. Gaeta, Neil G. R. Broderick, Alexander V. Sergienko, Arno Rauschenbeutel, Thomas Durt, Proc. of SPIE Vol. 9136, 913612 - (C) 2014 SPIE · CCC code: 0277-786X/14/\$18 · doi: 10.1117/12.2052207 
The unrealistically low number of modes used in numerical simulations ${ }^{15-16}$ is the main limitation of used numerical approaches as fine laser radiation properties (like deviations of intensity statistics from Gaussian, the probability of extreme events, mode correlations) could be calculated inaccurately.

In the present paper, we demonstrate a short RFL based on 12 meters of commercially available fiber. The laser generates a narrow spectrum of spectral width less than $200 \mathrm{pm}$ at $10 \mathrm{~W}$ of intra-cavity generation power ${ }^{17}$. The total number of generated longitudinal modes is $\sim 10^{4}$ so the laser properties could be directly modeled using the real number of modes. The measured laser intensity dynamics reveals indications of mode correlations.

\section{EXPERIMENT AND DISCUSSION}

The experimental setup is shown on Figure 1. The laser cavity is formed by $12 \mathrm{~m}$ of 980 -HP fibre with two highreflective FBGs. The gain in the system is provided by stimulated Raman scattering. Fiber Bragg gratings (FBGs) were used as cavity mirrors. Reflectivity of each FBG is around $98 \%$. The spectral width of FBGs is around $0.3 \mathrm{~nm}$ at the half maximum. To achieve the minimal cavity length under the available pumping, we have minimized intracavity losses in the cavity. To do this, we have written FBGs directly in the cavity fibre 980-HP to avoid intracavity losses at the splices. We found that maxima of the FBGs' reflection profiles are slightly unmatched, that increase the cavity losses. So we have used the temperature controller to alter the position of reflection spectrum of one of FBGs and perfectly match it with another one. As a pump laser the Ytterbium doped fibre laser operating at $1115 \mathrm{~nm}$ was used. The pump laser has output power of up to $12 \mathrm{~W}$. The pump wave optical spectrum width is $\sim 0.05 \mathrm{~nm}$. Pump laser was directly spliced to the Raman fiber laser optical cavity. We carefully checked that the generation of the Stokes wave does not influence the output performances of the pump laser. To measure directly the generation properties of the intra-cavity radiation, we have used the intracavity $1 \%$ coupler which was made in the same fibre which was used in the Raman fibre laser cavity. This allowed us to avoid a splice between coupler and cavity. All this measures resulted in the lowest possible laser length under the current pumping and cavity design. The designed laser has a generation threshold of $4.2 \mathrm{~W}$. The total intra-cavity power of the generated Stokes wave reaches $11 \mathrm{~W}$ at $12 \mathrm{~W}$ of pump.

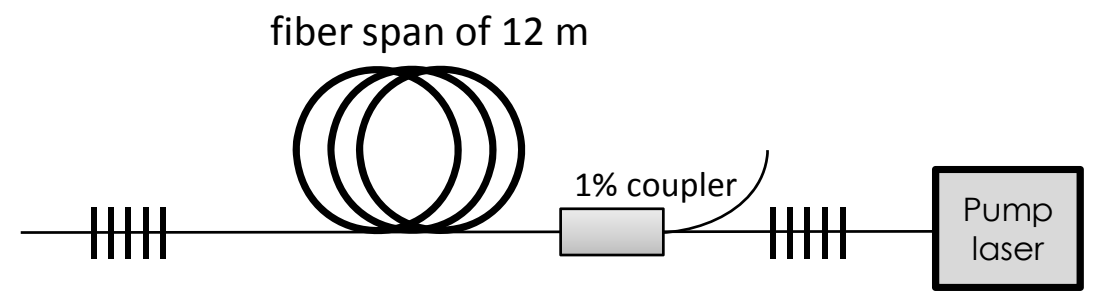

Fiber Bragg grating

Fiber Bragg grating

Figure 1. Experimental scheme of a short Raman fibre laser.

Despite the short cavity length, the generation spectrum is subject to nonlinear spectral broadening. Indeed, the generation spectrum is quite narrow at low pump power, near the threshold, Fig. 2(a). However, spectral broadening and pronounced spectral wings out of FBG bandwidth are generated at higher powers, Fig.2(b). The origin of spectral broadening in Raman fiber lasers is connected to the intense four-wave mixing processes which could be described in terms of wave turbulence ${ }^{89}$, . However, the spectrum width in the demonstrated short-cavity RFL is quite small comparing with longer lasers despite high generation power. Indeed, the spectral width is around $100 \mathrm{pm}$ only at low pump power, Fig. 2(a). This value is defined by the cross-phase modulation with the pump wave, so the generation spectrum acquires the spectral width being proportional to the spectral width of the used pump source ${ }^{8}$. The maximum observed spectrum width is below $200 \mathrm{pm}$, which is also remarkably low value for $11 \mathrm{~W}$ of generation power. To compare, the spectral width in conventional RFL having a long cavity is of order of $1 \mathrm{~nm}$ even at the smaller generation intracavity power, see, for example, ${ }^{18}$. 
(a)

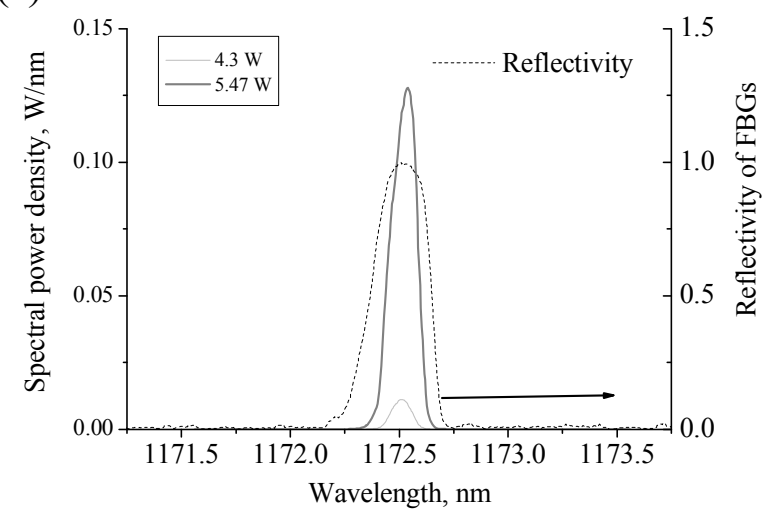

(b)

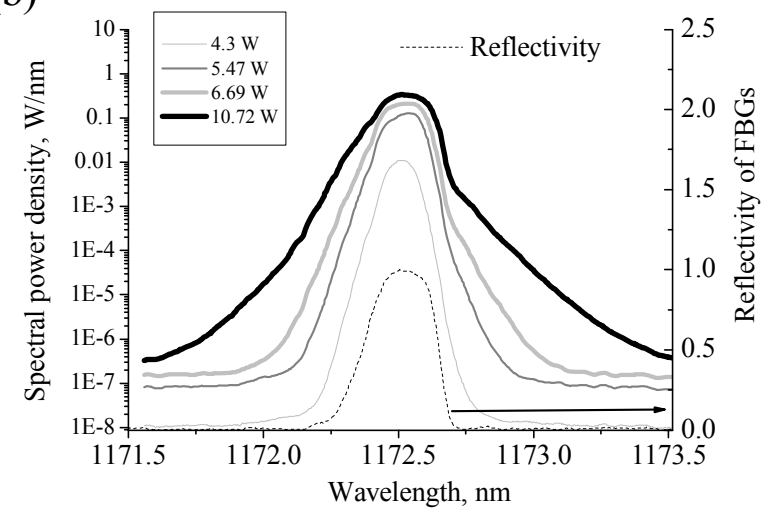

Figure 2. Generation spectrum and the spectrum of a fibre Bragg grating in linear (a) and logarithmic (b) scale.

The spectrum becomes broader with the generation power increase with almost linear dependence, Figure 3. It is known that in inlonger RFLs, the spectrum becomes broader as square-root of the generated power ${ }^{919}$. However, the spectral broadening law could be different in different systems: the spectral broadening law could be linear in short lasers. Indeed, Yb-doped fibre laser (YDFL) are subject to linear spectral broadening with the generation power increase due to non-linear self-modulation effect ${ }^{9}$. In the paper $^{9}$ an analytic model was developed in assumption of negligible dispersion-induced wave phase shift during the cavity roundtrip. The model is valid in the case of the generation spectrum width being much smaller than that of cavity mirrors. The model conditions are satisfied in demonstrated short RFL, so similar linear behaviour is observed.

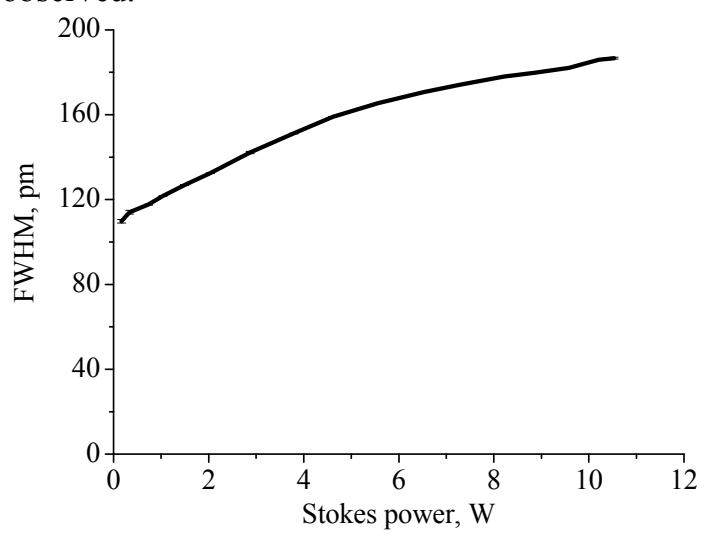

Figure 3. Full width at half-maximum of the generation spectrum.

Further, to reveal mode correlations in the generated radiation, we focus in our study on the temporal dynamics and statistical properties of the demonstrated short-cavity Raman fiber laser. In general, temporal dynamics and statistical properties of quasi-CW fibre lasers are of great interest in recent years. There are experimental indications that intensity statistics in quasi-CW fibre lasers could be non-gaussian revealing increased probability of extreme events in RFLs ${ }^{16,20}$ as well as non-Gaussian statistics in YDFL ${ }^{21}$. Moreover, recently it has been demonstrated that dark and grey solitons do really exist in the radiation of quasi-CW RFL operating in normal dispersion regime ${ }^{22}$.

One of benefits of short Raman fibre lasers is connected to challenges in numerical modelling of fibre lasers. To model the generation in a laser cavity, one has to solve the boundary value problem. This implies that the initial conditions for the field have to be applied over the numerical window being equal to the entire length of the laser resonator, and the field has to be further calculated over this temporal window. Typical RFL`s cavity length of $1 \mathrm{~km}$ corresponds to the $\sim 10 \mu$ s of the temporal window length. At the same time, the field has to be calculated with high enough resolution over the fast time being much smaller than the typical time of intensity fluctuations. The typical time of intensity fluctuations is inversely proportional to the spectrum width. Taking typical spectrum width at half maximum of $1 \mathrm{~nm}$, typical intensity fluctuations lasts for around $10 \mathrm{ps}$. Thus, practically, the field has to be calculated with a 
numerical step below $1 \mathrm{ps}$. This implies that a numerical grid of size of $\sim 10^{7}$ points has to be used in simulations that is practically impossible.

To make the numerical investigations feasible, it's worth working with shorter cavities. For instance in the presented short cavity RFL, the estimated round-trip for the $12 \mathrm{~m}$ of fiber span lasts for $120 \mathrm{~ns}$, thus to discern all the time dynamics features of $10 \mathrm{ps}$ duration, one should imply a grid of $\sim 10^{4}$ points that is practicable. That means that such a laser could be directly modelled using realistic number of longitudinal modes or temporal window. It is of interest to compare predictions of different models for laser statistical properties and mode correlations with experimental data to highlight the restrictions of used models. The results of this investigation will be published elsewhere.
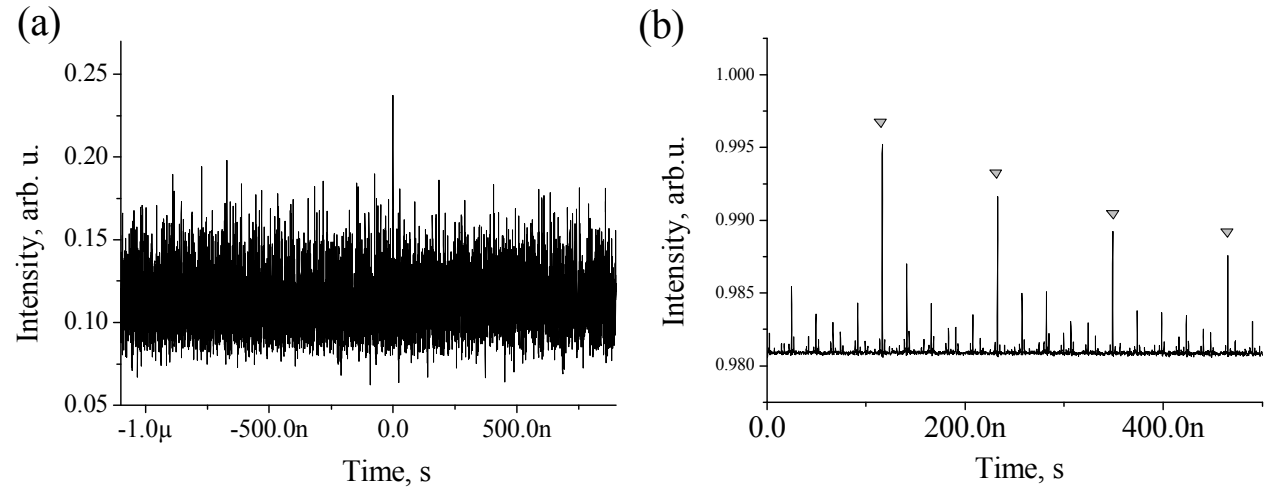

Figure 4. (a) Typical time dynamics measured in the short Raman fiber laser. (b) autocorrelation function $K(\tau)$. Triangles label the roundtrip spaced peaks.

Further, we focus on the experimental indications of mode correlations. We experimentally measure time dynamics, Fig. 4(a). Time resolution of the measurements was around $2 \mathrm{GHz}$ or $0.5 \mathrm{~ns}$ that means sufficient of the fast events lasting around $1 \mathrm{~ns}$. The intensity dynamics looks very sporadic, Fig. 4(a), so it is hardly to find there any indications of mode correlations. However, much clear picture can be obtained if special technique of intensity dynamics measurements is implemented. Namely, we measure an averaged time dynamics $S(\tau)$ taking into account only those events, which have an amplitude higher than some trigger level. To do that we use a trigger of the oscilloscope which is set to some value $I_{\text {set. }}$. Only traces comprising events $I(t)>I_{\text {set }}$ contributes to the average time dynamics $S(\tau)$. The trigger level $I_{\text {set }}$ is typically chosen to be 2-3 times higher than average intensity level $\langle I(t)\rangle_{t}$. Mathematically, we measure the following function of the intensity dynamics $I(t)$ :

$$
\mathrm{S}(\tau)=\left\langle I(t+\tau) \cdot H\left(\frac{I(t)}{I_{s e t}}-1\right)\right\rangle_{t}
$$

where $H(x)$ is Heaviside function. Averaging is performed over different realizations in time. As a result, we reveal typical time dynamics before and after an event with intensity $I>I_{\text {set }}$, Fig. 5a. If correlations do exist, the triggered intense event should live a considerable time, oscillating with intermode frequency $v_{r t}=c / 2 L$ and therefore appear every roundtrip period $T_{r t}=\frac{1}{v_{r t}}=120 \mathrm{~ns}$. These oscillations are directly revealed on Fig. 4b. Correlations remain for approximately 10 roundtrips, Fig. 5a, corresponding events are marked with triangles. After that phase relations are changed, and, interestingly, some small anti-correlations do appear, leading to destructive interference of the modes and resulting in dips in the trace instead of intense peaks, figure 5(a) (inset). Besides, there are some additional peaks located at detunings not equal to integer number of roundtrips, Fig. 5(a). Note, that the similar picture can be found in the common intensity autocorrelation function $K(\tau)=\frac{\langle I(t+\tau) I(t)\rangle_{t}}{\left\langle I^{2}(t)\right\rangle_{t}}$, see Fig.4(b).

The similar dynamics was experimentally observed in a short ytterbium-doped fibre laser with a narrow spectrum operating well above the generation threshold (see laser details in the pape ${ }^{21}$ ). On the figure $5(\mathrm{~b})$ derived function $K(\tau)=\frac{\langle I(t+\tau) I(t)\rangle_{t}}{\left\langle I^{2}(t)\right\rangle_{t}}$ is shown. Here the anti-correlations are even more pronounced. In addition, region of correlation revives after the region of anti-correlation. 
(a)

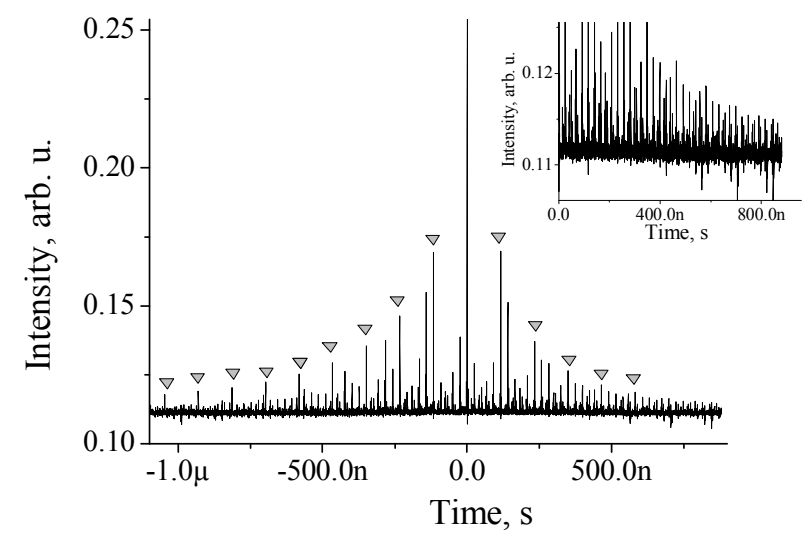

(b)

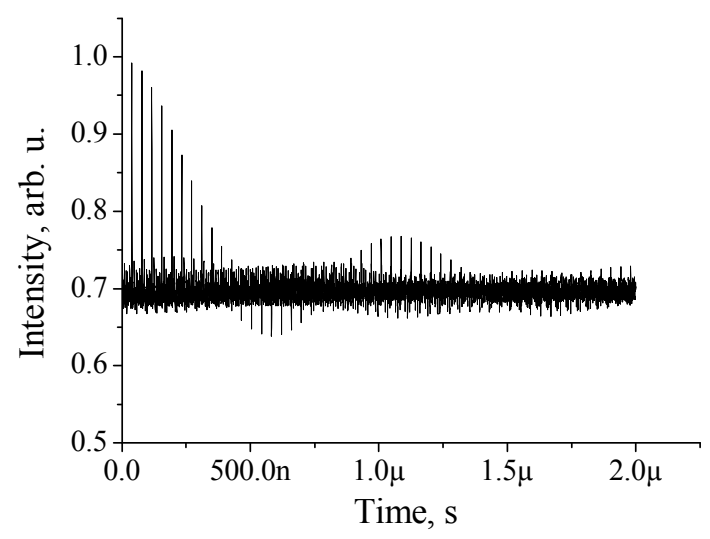

Figure 5. Mode correlations in the radiation of a quasi-CW fibre laser. (a) $\mathbf{S}(\boldsymbol{\tau})$ for the short cavity RFL. Inset - the same trace zoomed to point out anti-correlations. (b) autocorrelation function for the short cavity YDFL.

\section{CONCLUSION}

We have presented a short Raman fibre laser based on $12 \mathrm{~m}$ only of commercially available fibre (980-HP). The width of the generation spectrum experiences almost linear growth with the generated power due to nonlinear broadening and varies from $100 \mathrm{pm}$ to $200 \mathrm{pm}$, that is sufficiently narrower that the typical spectral width of the longer Raman fiber lasers. As the spectrum is narrow and the laser cavity is short, relatively small number (around 10000 ) of longitudinal modes is generated. We have performed experimental investigation of spectral modes correlations by measuring an intensity dynamics averaged over the events with the intensity higher than an intensity of a trigger. It is found that longliving (tens roundtrips) intense event are generated. The interchange between positive and negative interference between the modes is found. The similar behaviour has been observed in time dynamics of $4 \mathrm{~m}$ long $\mathrm{Yb}$ doped fiber laser.

\section{REFERENCES}

[1] Turitsyn, S.K., Ania-Castanon, J.D., Babin, S.A., Karalekas, V., Harper, P., Churkin, D. V., Kablukov, S., El-Taher, A.E., Podivilov, E. V., et al., "270-km Ultralong Raman Fiber Laser,” Physical Review Letters 103(13), 133901 (2009).

[2] Ania-Castanon, J.D., Ellingham, T., Ibbotson, R., Chen, X., Zhang, L., and Turitsyn, S., "Ultralong Raman Fiber Lasers as Virtually Lossless Optical Media," Physical Review Letters 96(2), 023902 (2006).

[3] Alcón-Camas, M., and Ania-Castanon, J.D., "RIN transfer in 2nd-order distributed amplification with ultralong fiber lasers," Optics Express 18(23), 23569 (2010).

[4] Nicholson, J.W., Yan, M.F., Wisk, P., Fleming, J., DiMarcello, F., Monberg, E., Taunay, T., Headley, C., and DiGiovanni, D.J., "Raman fiber laser with $81 \mathrm{~W}$ output power at $1480 \mathrm{~nm}$," Optics Letters 35(18), 30693071 (2010).

[5] Feng, Y., Taylor, L.R., and Calia, D.B., "150 W highly-efficient Raman fiber laser," Optics Express 17(26), 23678-83 (2009).

[6] Zhao, Y., and Jackson, S.D., "Highly efficient first order Raman fibre lasers using very short Ge-doped silica fibres," Optics Communications 253(1-3), 172-176 (2005).

[7] Georgiev, D., Gapontsev, V.P., Dronov, a G., Vyatkin, M.Y., Rulkov, a B., Popov, S. V, and Taylor, J.R., "Watts-level frequency doubling of a narrow line linearly polarized Raman fiber laser to $589 \mathrm{~nm}$.," Optics express 13(18), 6772-6 (2005).

[8] Babin, S.A., Churkin, D. V., Ismagulov, A.E., Kablukov, S.I., and Podivilov, E. V., "Turbulenceinduced square-root broadening of the Raman fiber laser output spectrum," Optics Letters 33(6), 633-635 (2008). 
[9] Babin, S.A., Churkin, D. V., Ismagulov, A.E., Kablukov, S.I., and Podivilov, E. V., "Four-wavemixing-induced turbulent spectral broadening in a long Raman fiber laser," Journal of the Optical Society of America B 24(8), 1729 (2007).

[10] Siekiera, A., Engelbrecht, R., Nothofer, A., and Schmauss, B., "Short 17-cm DBR Raman Fiber Laser With a Narrow Spectrum,” IEEE PHOTONICS TECHNOLOGY LETTERS 24(2), 107-109 (2012).

[11] Westbrook, P.S., Abedin, K.S., Nicholson, J.W., Kremp, T., and Porque, J., "Raman fiber distributed feedback lasers," Optics letters 36(15), 2895-7 (2011).

[12] Chamorovskiy, a, Rautiainen, J., Lyytikäinen, J., Ranta, S., Tavast, M., Sirbu, a, Kapon, E., and Okhotnikov, O.G., "Raman fiber laser pumped by a semiconductor disk laser and mode locked by a semiconductor saturable absorber mirror.," Optics letters 35(20), 3529-31 (2010).

[13] Castellani, C.E.S., Kelleher, E.J.R., Popa, D., Hasan, T., Sun, Z., Ferrari, a C., Popov, S. V, and Taylor, J.R., “CW-pumped short pulsed $1.12 \mu \mathrm{m}$ Raman laser using carbon nanotubes,” Laser Physics Letters 10(1), 015101 (2013).

[14] Turitsyna, E.G., Smirnov, S. V, Sugavanam, S., Tarasov, N., Shu, X., Babin, S.A., Podivilov, E. V., Churkin, D. V., Falkovich, G., et al., "Suppl. The laminar-turbulent transition in a fibre laser," Nature Photonics 7(10), 783-786 (2013).

[15] Churkin, D. V., Smirnov, S. V, and Podivilov, E. V., "Statistical properties of partially coherent CW fiber lasers," Optics Letters 35(19), 3288-90 (2010).

[16] Randoux, S., and Suret, P., "Experimental evidence of extreme value statistics in Raman fiber lasers," Optics letters 37(4), 500-2 (2012).

[17] Vatnik, I.D., Gorbunov, O.A., and Churkin, D. V., "Narrow-band generation and mode correlations in a short Raman fibre laser," Laser Physics 24(2), 025103 (2014).

[18] Huang, S., Feng, Y., Shirakawa, A., and Ueda, K., "Generation of 10.5 W, 1178 nm Laser Based on Phosphosilicate Raman Fiber Laser," Japanese Journal of Applied Physics 42(Part 2, No. 12A), L1439-L1441 (2003).

[19] Suret, P., Walczak, P., and Randoux, S., "Transient buildup of the optical power spectrum in Raman fiber lasers," Optics Express 21(2), 2331-2336 (2013).

[20] Churkin, D. V., Gorbunov, O.A., and Smirnov, S. V, "Extreme value statistics in Raman fiber lasers," Optics Letters 36(18), 3617-3619 (2011).

[21] Bednyakova, A.E., Gorbunov, O.A., Politko, M.O., Kablukov, S.I., Smirnov, S. V., Churkin, D. V., Fedoruk, M.P., and Babin, S.A., "Generation dynamics of the narrowband Yb- doped fiber laser," Optics Express 21(7), 8177-8182 (2013).

[22] Turitsyna, E.G., Smirnov, S. V., Sugavanam, S., Tarasov, N., Shu, X., Babin, S. a., Podivilov, E. V., Churkin, D. V., Falkovich, G., et al., "The laminar-turbulent transition in a fibre laser," Nature Photonics 7(10), 783-786 (2013). 